\title{
The interferon paradox: can inhibiting an antiviral mechanism advance an HIV cure?
}

\author{
Steven G. Deeks, ${ }^{1}$ Pamela M. Odorizzi, ${ }^{1}$ and Rafick-Pierre Sekaly ${ }^{2}$
}

'UCSF, San Francisco, California, USA. ² Case Western Reserve University, Cleveland, Ohio, USA.

\begin{abstract}
While antiretroviral therapy (ART) has improved the quality of life and increased the life span of many HIV-infected individuals, this therapeutic strategy has several limitations, including a lack of efficacy in fully restoring immune function and a requirement for life-long treatment. Two studies in this issue of the $\mathrm{JCl}$ use a humanized mouse model and demonstrate that type I interferon (IFN) is induced early during HIV infection and that type I IFNassociated gene signatures persist, even during ART. Importantly, blockade of type I IFN improved immune function, reduced the HIV reservoir, and caused a delay in viral rebound after ART interruption. Together, these two studies support further evaluation of IFN blockade as a supplement to ART.
\end{abstract}

\section{Current challenges for} antiretroviral therapy

Those HIV-infected individuals who are able to access, tolerate, and adhere to combination antiretroviral therapy (ART) can expect to have a near-normal life span, particularly if ART is initiated during acute infection, before the virus causes significant damage to the immune system. Despite the success of ART, there are two wellrecognized limitations of current strategies. First, due to resource limitations and/or treatment toxicities, many of the 37 million HIV-infected individuals worldwide are unable to remain on therapy indefinitely. Second, the vast majority of individuals who start therapy do so during more advance stages of the disease, after extensive damage to the immune system has already occurred. Chronic immune dysfunction during ART has been associated with the development of a number of non-AIDS-related complications, including cardiovascular disease and cancer. As lifetime treatment poses a variety of challenges, there is intense interest in developing therapeutic interventions that result in sustained control of the virus in the absence of therapy (a remission or cure; ref. 1). There is also great interest in thera- pies that reverse the chronic inflammation that appears to be central to development of immune dysfunction and non-AIDS morbidity. Therapies that address both of these recognized limitations would be particularly exciting advances.

\section{Type I IFN - beneficial or detrimental?}

Long-term treatment of HIV infection results in a state of chronic inflammation. Multiple factors contribute to this inflammatory state, including HIV production, irreversible loss of mucosal integrity and exposure to microbes, and an excess burden of other chronic pathogens, particularly CMV. Central to each of these inflammation-inducing pathways is the sustained upregulation of type I interferons (IFNs), which in turn triggers the transcription of a multitude of IFN-stimulated genes (ISGs) that regulate the function of $\mathrm{T}$ cells and other components of the immune system. During acute infection, the type I IFN signaling is clearly beneficial. Indeed, experimental inhibition of type I IFN during acute SIV infection causes poor virus control, accelerated loss of immune function, and death (2). During chronic infection,

Related Articles: pp. 260 and 269

Conflict of interest: S.G. Deeks receives research support from Merck, Gilead, and ViiV Healthcare.

Reference information: / Clin Invest. 2017;127(1):103-105. doi:10.1172/JCI91916. 


\section{IFN blockade during chronic HIV}

Zhen et al. and Cheng et al. demonstrated that blockade of the IFN receptor during the chronic phase of infection reduces the levels of $\mathrm{T}$ cell activation, reduces expression of the inhibitory receptors PD-1 and TIM-3, and improves cytokine production by CD $8^{+} \mathrm{T}$ cells $(18,19)$. Interestingly, IFN blockade during chronic infection also resulted in lower levels of HIV replication, presumably due to improved $\mathrm{CD}^{+} \mathrm{T}$ cell function and/or reduced frequencies of activated $\mathrm{CD} 4^{+} \mathrm{T}$ cells, which are the preferred target cell for spreading HIV.

Perhaps the most fascinating observations from these studies are that type I IFN blockade during ART administration markedly reduced the frequency of cells harboring replication-competent HIV, the so-called "reservoir" $(18,19)$, and caused a delayed rebound of viremia after ART was discontinued (19). The mechanism(s) that underlie these effects, however, are not clear from these mouse studies. Based in part on a series of observations that have been made in humans by our group and others, we propose that the beneficial effect of IFN blockade on the reservoir could be due to at least three mechanisms $(20,21)$. First, as $\mathrm{CD}^{+} \mathrm{T}$ cell activation drives virus production and propagation of infection, it may be that reduced $\mathrm{T}$ cell activation following IFN receptor blockade decreases the pool of cells that are actively producing virus and/or that are susceptible to infection, leading to reduced virus spread. Second, as $\mathrm{CD} 4^{+} \mathrm{T}$ cell proliferation (both antigen driven and homeostatic) and long-term survival both contribute to HIV reservoir persistence, it is reasonable to postulate that IFN blockade-mediated alterations in these pathways would lead to gradual depletion of the reservoir over time. Finally, and we believe most importantly, IFN blockademediated improvements in $\mathrm{CD}^{+} \mathrm{T}$ cell function would be expected to result in accelerated clearance of virus-producing cells during ART and improved control after ART interruption. Concurrent with this, temporary increases in viral load (viral load blipping) following IFN blockade suggest a release of pathways that actively suppress low-level replication and/or latency, which, in conjunction with improved $\mathrm{CD}^{+} \mathrm{T}$ cell function, may lead to a successful "shock-and-kill” approach.

\section{Concluding remarks}

Chronic type I IFN signaling is central to the pathogenesis of a number of other diseases, including systemic lupus erythematosus, rheumatoid arthritis, and perhaps cancer. This has led to the development of several clinical interventions that include the use of antibodies to block IFNs and their downstream target genes to treat these autoimmune diseases (22). Emerging phase II/III clinical trial data suggest that these pathways can be inhibited therapeutically in an effective and safe manner $(23,24)$. Should these agents continue to show a favorable safety profile, we believe that efforts should be made to move these interventions into the HIV cure arena. Based on the work by Cheng et al. (19) and Zhen et al. (18), one might predict that administration of an antibody that blocks the type I IFN signaling in ART-suppressed HIV disease might (a) improve immunity by decreasing immune hyperactivation, decreasing expression of PD-1 and other negative regulators, and enhancing $\mathrm{CD}^{+} \mathrm{T}$ cell functionality; (b) curb non-AIDS morbidities associated with chronic inflammation; and (c) decrease the size and/or stability of the HIV reservoir. Collectively, these effects would lead to accelerated clearance of virus-producing cells during ART. The timing and duration of administration of IFN blockade will be critical in moving this approach forward in the clinic, as the level of viral suppression induced by ART, the degree of CD4 ${ }^{+}$ $\mathrm{T}$ cell recovery, and the severity of $\mathrm{CD} 8^{+}$ $\mathrm{T}$ cell exhaustion will be critical cofactors. Combination strategies to further enhance $\mathrm{CD}^{+} \mathrm{T}$ cell functionality or improve $\mathrm{T}$ cell migration into HIV "sanctuaries" may synergize to provide an even more robust "cure" strategy. Ultimately, this approach has the potential to improve control of HIV in the absence of ART and further the goal of developing a cure for HIV.

\section{Acknowledgments}

The investigators acknowledge the Delaney AIDS Research Enterprise (DARE) collaboration (UM1AI126611) and the amfAR Institute for HIV Cure Research (amfAR 109301).

Address correspondence to: Steven G. Deeks, 995 Potrero Avenue, San Francisco, California 94114, USA. Phone: 415.476.4082, ext. 404; E-mail: Steven.Deeks@ucsf.edu.
1. Deeks SG, et al. International AIDS Society global scientific strategy: towards an HIV cure 2016. Nat Med.2016;22(8):839-850.

2. Sandler NG, et al. Type I interferon responses in rhesus macaques prevent SIV infection and slow disease progression. Nature. 2014;511(7511):601-605.

3. Hardy GA, et al. Interferon- $\alpha$ is the primary plasma type-I IFN in HIV-1 infection and correlates with immune activation and disease markers. PLoS One. 2013;8(2):e56527.

4. Favre D, et al. Tryptophan catabolism by indoleamine 2,3-dioxygenase 1 alters the balance of TH17 to regulatory T cells in HIV disease. Sci Transl Med. 2010;2(32):32ra36.

5. Le Saout C, et al. Chronic exposure to type-I IFN under lymphopenic conditions alters CD4 T cell homeostasis. PLoS Pathog. 2014;10(3):e1003976.

6. Fernandez S, et al. CD4 ${ }^{+}$T-cell deficiency in HIV patients responding to antiretroviral therapy is associated with increased expression of interferon-stimulated genes in $\mathrm{CD}^{+}{ }^{+} \mathrm{T}$ cells. J Infect Dis. 2011;204(12):1927-1935.

7. Dunham RM, et al. Discordance between peripheral and colonic markers of inflammation during suppressive ART. J Acquir Immune Defic Syndr. 2014;65(2):133-141.

8. Tenorio AR, et al. Soluble markers of inflammation and coagulation, but not T-cell activation, are predictors of non-AIDS-defining morbid events during suppressive antiretroviral treatment. J Infect Dis. 2014;210(8):1248-1259.

9. Hunt PW, et al. Gut epithelial barrier dysfunction and innate immune activation predict mortality in treated HIV infection. J Infect Dis. 2014;210(8):1228-1238.

10. Lederman MM, et al. Immunologic failure despite suppressive antiretroviral therapy is related to activation and turnover of memory CD4 cells. J Infect Dis. 2011;204(8):1217-1226.

11. Catalfamo M, et al. CD4 and CD8 T cell immune activation during chronic HIV infection: roles of homeostasis, HIV, type I IFN, and IL-7. J Immunol. 2011;186(4):2106-2116.

12. Mudd JC, et al. Impaired T-cell responses to sphingosine-1-phosphate in HIV-1 infected lymph nodes. Blood. 2013;121(15):2914-2922.

13. Manion $\mathrm{M}$, et al. Interferon-alpha administration enhances $\mathrm{CD} 8^{+} \mathrm{T}$ cell activation in HIV infection. PLoS One. 2012;7(1):e30306.

14. Stoddart CA, Keir ME, McCune JM. IFN$\alpha$-induced upregulation of CCR 5 leads to expanded HIV tropism in vivo. PLoS Pathog. 2010;6(2):e1000766.

15. Odorizzi PM, Wherry EJ. Immunology. Science. 2013;340(6129):155-156.

16. Wilson EB, et al. Blockade of chronic type I interferon signaling to control persistent LCMV infection. Science. 2013;340(6129):202-207.

17. Teijaro JR, et al. Persistent LCMV infection is controlled by blockade of type I interferon signaling. Science. 2013;340(6129):207-211.

18. Zhen A, et al. Targeting type I interferonmediated activation restores immune function in chronic HIV infection. JClin Invest. 2017;127(1):260-268. 
19. Cheng L, et al. Blocking type I interferon signaling enhances T cell recovery reduces HIV-1 reservoirs. JClin Invest. 2017;127(1):269-279.

20. Klatt NR, Chomont N, Douek DC, Deeks SG. Immune activation and HIV persistence: implications for curative approaches to HIV infection. Immunol Rev. 2013;254(1):326-342.

21. Katlama C, et al. Barriers to a cure for HIV: new ways to target and eradicate HIV-1 reservoirs. Lancet. 2013;381(9883):2109-2117.

22. Oon S, Wilson NJ, Wicks I. Targeted therapeutics in SLE: emerging strategies to modulate the interferon pathway. Clin Transl Immunology. 2016;5(5):e79.

23. Kalunian KC, et al. A phase II study of the efficacy and safety of rontalizumab (rhuMAb interferon- $\alpha$ ) in patients with systemic lupus erythematosus (ROSE). Ann Rheum Dis. 2016;75(1):196-202.

24. Khamashta M, et al. Sifalimumab, an anti-interferon- $\alpha$ monoclonal antibody, in moderate to severe systemic lupus erythematosus: a randomised, double-blind, placebo-controlled study. Ann Rheum Dis. 2016;75(11):1909-1916. 